\title{
Generating derivative structures from multilattices: Algorithm and application to hcp alloys
}

Gus L. W. Hart

gus.hart@gmail.com

Rodney W. Forcade

Follow this and additional works at: https://scholarsarchive.byu.edu/facpub

Part of the Astrophysics and Astronomy Commons, and the Physics Commons

\section{Original Publication Citation}

Gus L. W. Hart and Rodney W. Forcade, "Generating derivative structures from multilattices: Application to hcp alloys," Phys. Rev. B8 1412 (July 29). The original article may be found here: http://prb.aps.org/abstract/PRB/v8/i1/e1412

\section{BYU ScholarsArchive Citation}

Hart, Gus L. W. and Forcade, Rodney W., "Generating derivative structures from multilattices: Algorithm and application to hcp alloys" (2009). Faculty Publications. 127.

https://scholarsarchive.byu.edu/facpub/127 


\title{
ş \\ Generating derivative structures from multilattices: Algorithm and application to hcp alloys
}

\author{
Gus L. W. Hart ${ }^{1}$ and Rodney W. Forcade ${ }^{2}$ \\ ${ }^{1}$ Department of Physics \& Astronomy, Brigham Young University, Provo, Utah 84602, USA \\ ${ }^{2}$ Department of Mathematics, Brigham Young University, Provo, Utah 84602, USA \\ (Received 10 April 2009; revised manuscript received 6 July 2009; published 31 July 2009)
}

\begin{abstract}
We present an algorithm for generating all derivative superstructures of a nonprimitive parent lattice. The algorithm has immediate application in important materials design problems such as modeling hexagonalclose-packed (hcp) alloys. Extending the work of Hart and Forcade [Phys. Rev. B 77, 224115 (2008)] (which applies only to Bravais lattices), this approach applies to arbitrary multilattices. The algorithm enumerates superlattices and atomic configurations using permutation groups rather than direct geometric comparisons. The key concept is to use the quotient group associated with each superlattice to determine all unique atomic configurations. The algorithm is very efficient; the run time scales linearly with the number of unique structures found. We demonstrate the algorithm in the important case of hcp-derived superstructures. In the list of enumerated hexagonal-close-packed derivative superstructures, we predict several as-yet-unobserved structures as likely candidates for new intermetallic prototypes.
\end{abstract}

DOI: 10.1103/PhysRevB.80.014120

PACS number(s): 61.50.Ah, 61.66.Dk, 61.90.+d, 61.50.Nw

\section{INTRODUCTION}

Many of the ordered structures observed in intermetallic and semiconductor alloys are derived from a parent latticethey are derivative superstructures. To search for new structures, it is useful to have a list of all possible superstructures. Generating all combinatorially distinct structures is relatively simple, but removing those structures that are geometrically equivalent from this exhaustive list is more difficult. Previous enumeration algorithms are inefficient because they use geometric comparison to remove duplicate structures. The running time of our approach scales linearly in the number of unique structures identified. Our approach relies on group theory and an integer representation of the superstructures.

In this paper, we generalize the group-theoretic method ${ }^{1}$ so that it can be applied to cases where the parent is not a Bravais (i.e., simple) lattice but a multilattice. A multilattice is a set of atomic sites that do not constitute a lattice because the points of a multilattice are not all translationally equivalent. That is, the primitive unit cell contains more than one lattice point. More formally, a multilattice $M$ is a union of translates of a lattice $L$ offset by a set of fractional translations $D$. A two-dimensional example of a multilattice is shown in Fig. 1. Three-dimensional examples include the diamond structure and the hexagonal-close-packed (hcp) structure.

The two important aspects of our method are a grouptheoretic approach and an integer representation of derivative structures. These key points simplify the implementation of an algorithm and facilitate an $\mathcal{O}(N)$ scaling. Additionally, the integer representation for derivative structures streamlines the cluster expansion methodology and reduces computer memory storage requirements. With the reduced memory requirements, lattice Monte Carlo simulations with a billion atoms are now possible. ${ }^{2}$

\section{DERIVATIVE SUPERSTRUCTURES}

A derivative superstructure is an atomic configuration of a lattice whose periodicity is determined by a superlattice. ${ }^{3}$
Figure 2 illustrates the superstructure idea with two examples - two fictional crystals - in two dimensions. The parent lattice is the square lattice shown on the left [Fig. 2(a)]. The superlattices of Figs. 2(b) and 2(c) are integer multiples of the square lattice. The periodicity of each superstructure is indicated by the dotted lines. The atoms of each superstructure lie on the parent lattice points, ${ }^{4}$ but the periodicity of the atomic configurations matches the periodicity of the superlattices.

The preceding examples are two dimensional, but many real three-dimensional examples of derivative superstructures are found in metal and semiconductor alloys. Two examples of intermetallic derivative structures are shown in Fig. 3. The $\mathrm{Cu}_{3} \mathrm{Au}$ structure, Strukturbericht $L 1_{0}$ (upper right), is derived from an fcc parent lattice (upper left). The $\mathrm{Ni}_{3} \mathrm{Sn}$ structure, Strukturbericht $D 0_{19}$, (lower right) is derived from an hcp parent multilattice. These two structures appear frequently in many industrially important alloys. A compendium of fcc-, bcc-, and hcp-derived superstructures, taken from the experimental literature, is given by Sluiter in Ref. 5.

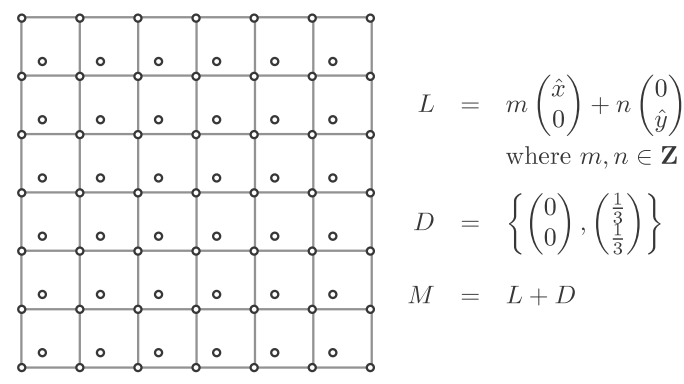

FIG. 1. A two-dimensional example of a multilattice. In each unit cell there are two atomic sites. Because this set of atomic sites cannot be defined by just two vectors (i.e., the unit cell only), it is not a lattice in the proper sense. Rather we refer to it as a multilattice or, more specifically, as a two-lattice: it is two square lattices superimposed-one placed at the origin and another translated slightly in the direction $\hat{x}+\hat{y}$. The origins of each lattice constitute the $D$ set, $(0,0)$, and $\left(\frac{1}{3}, \frac{1}{3}\right)$ in this case. 


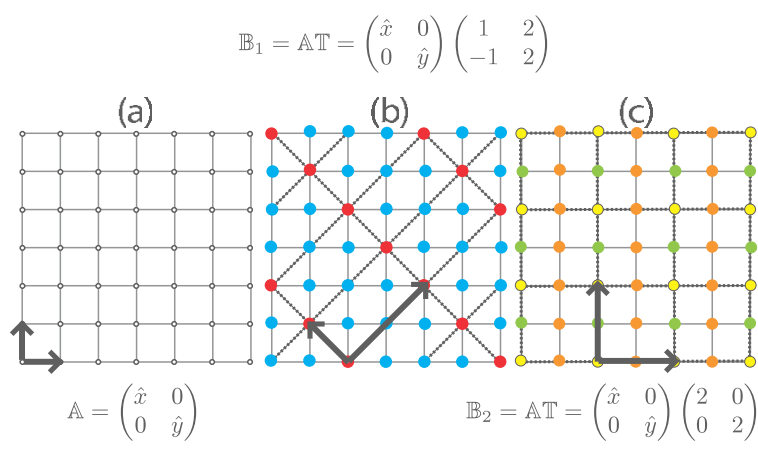

FIG. 2. (Color online) (a) A square parent lattice and (b) and (c) two derivative structures. The dotted lines in the superstructures show the superlattices, which are multiples of the parent lattice. That is, the dotted lines indicate the unit cell of the superstructure. The lattice vectors are indicated by arrows. The superstructure lattice vectors are integer combinations of the parent cell lattice vectors. In the superstructures, the atoms (colored circles) lie on the lattice points of the original parent lattice and have a periodicity that matches the superlattice.

Derivative structures are common in intermetallic systems and semiconductors. Therefore, it is useful to enumerate derivative structures whenever the physical observable of interest depends directly on the atomic configuration. Given an exhaustive list of all derivative structures, we can quickly find the structure that optimizes a target property.

\section{GROUP-THEORETIC APPROACH}

The motivation for addressing this problem is applications in physics, chemistry, and materials science. However, for convenience it is simpler to refer to the structures and their enumeration in mathematical terms, using words such as lat-

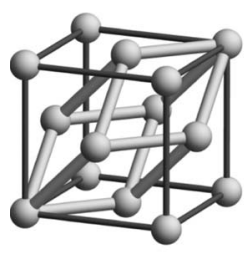

fcc

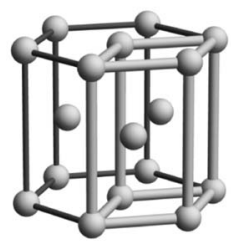

hcp

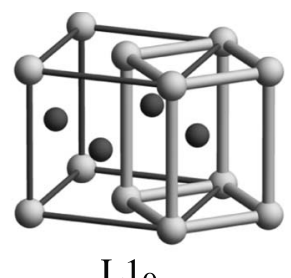

$\mathrm{L} 1_{0}$

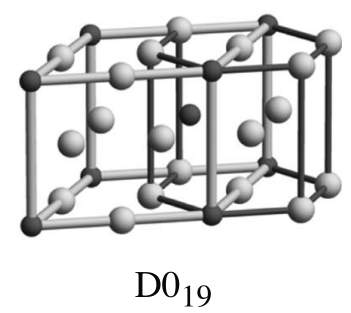

FIG. 3. Two real examples of three-dimensional superstructures. The $L 1_{0}$ structure (top right) is a doubled superstructure derived from the fcc lattice (top left). The $D 0_{19}$ structure (bottom right) is a quadrupled superstructure derived from an hcp parent multilattice (bottom left). tices and labelings rather than unit cells, atomic sites, and atom types. In mathematical terms, the problem of enumerating superstructures is a combinatorial one. The lattice is defined by a set of basis vectors, and a color or label is assigned to each lattice point. The labeling must be periodic with respect to a tiling of the lattice by a superlattice. Of course there are an infinite number of such arrangements, but one may generate them systematically by starting with the smallest tilings first.

The group-theoretical approach we employ (see Ref. 1) takes advantage of well-known properties of integer matrices (discussed in detail in the following section) and the properties of permutation groups. Any derivative superlattice is represented by an integer matrix. Take, for example, the superstructures of Fig. 2. The parent lattice is shown on the left; the basis vectors are shown and the corresponding matrix $\mathrm{A}$ is $\left(\begin{array}{ll}\hat{x} & 0 \\ 0 & \hat{y}\end{array}\right)$. For the structure shown in the middle panel, the superlattice can be defined as $\mathrm{B}=\mathrm{AT}$, where $\mathrm{T}=\left(\begin{array}{cc}-1 & 2 \\ 1 & 2\end{array}\right)$; clearly the superlattice vectors are integer linear combinations of the column vectors of $\mathrm{A}$ (because the entries of $\mathrm{T}$ are integer). The superlattice is four times larger than the parent; each tile contains four points of the parent lattice. The structure shown on the right, part (c), is also four times larger than the parent, but the tiling has a different shape than the middle one.

The integer representation of the superlattice is unique using Hermite normal form (HNF), defined below. Thus, generating all HNF matrices is equivalent to generating all possible superlattices. ${ }^{6}$ We settle for generating all HNF matrices up to a maximum determinant that meets or exceeds any practical application.

Reducing an HNF matrix to Smith normal form (SNF) defines a composite group that conveniently represents the translation symmetries. ${ }^{7}$ Each element of this finite composite group represents an infinite set of lattice points that must all receive the same label (such sets are called cosets). Using the group representation, the symmetrically distinct labelings of points inside the superlattice can be readily identified. While using a group-theoretic representation for the problem may seem unduly abstract, it leads to an extremely efficient algorithm.

Before discussing the algorithm in detail, we use the example of Fig. 4 to demonstrate how symmetry operations of a multilattice can be considered merely as permutations of the labels. The attractive feature of the algorithm is that geometric symmetries can be represented as permutations of the labels. The permutations come directly from the group defined by the SNF of the superlattice.

Figure 4(a) shows that a multilattice is constructed from a simple lattice by using a $D$ set that contains more than just the point $(0,0)$. Part (b) shows that a multiple of the multilattice defines a superlattice. Part (c) illustrates that each translated copy of the superlattice (each translate) receives a single label.

The second row of Fig. 4 demonstrates how the superlattice points are permuted by a symmetry operation of the multilattice. The points of the original superlattice are reflected about the line $x+y=0$ and then shifted by a member of the $D$ set, $\left(\frac{1}{3}, \frac{1}{3}\right)$. (In general, a symmetry of the multilat- 

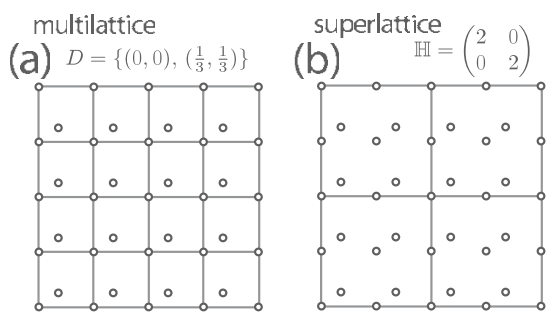

same label for each (C) member of a coset

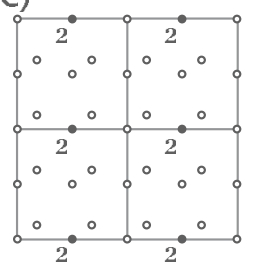

(d)

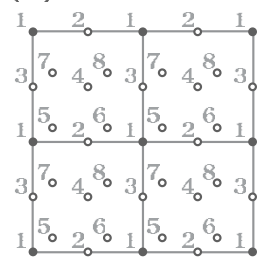

(e)

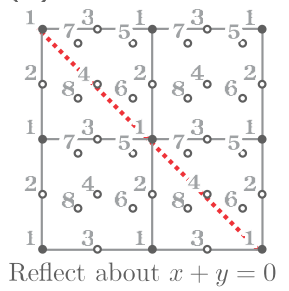

(f)
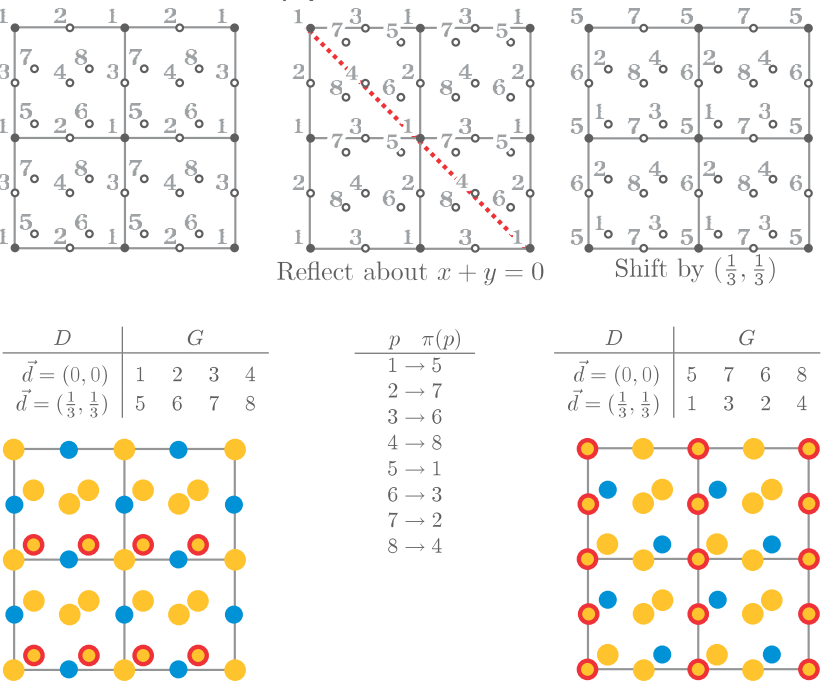

FIG. 4. (Color online) An example demonstrating that the geometric equivalence of multilattice labelings can be expressed as a permutation group. (a) is a multilattice, a square lattice with two points in the $D$ set: $(0,0)$ and $\left(\frac{1}{3}, \frac{1}{3}\right)$. (b) is a superlattice derived from (a) using the given transformation matrix $\mathbb{H}$. (c) shows that if any point is labeled, all equivalent points (translates) must have the same label. (The set of such points is a coset.) (d)-(f) show how lattice points are permuted by a symmetry operation of the multilattice, an orthogonal transformation followed by a fractional translation. (d) is the starting configuration. (e) shows how the points are moved by a reflection about the $x+y=0$ line. (f) shows the reflected points of (e) translated so the permuted points are now coincident with the original superlattice. This symmetry operation permutes the elements of the first $D \times G$ table to form the second table (rows reversed, second and third columns exchanged). The middle table depicts the permutation mapping, $\pi$, that maps each point, $p$, of the superlattice to another. The bottom left picture shows one particular but arbitrary labeling of the multilattice; the bottom right shows how this labeling is permuted by the symmetry operation. The second labeling is not identical to the first but it is equivalent.

tice comprises a rotation/reflection and a fractional shift; the shift can always be chosen to be a member of the $D$ set. See the Appendix.) After the symmetry operation, all of the points of the transformed superlattice are coincident with the original points, but they have been rearranged, that is, permuted.

As a specific illustration, the labeled superlattice shown at the bottom left of Fig. 4 is transformed into the labeled superlattice at the bottom right. These two labelings are not identical but they are equivalent labelings. Representing equivalent labelings as permutations of the group defined by the SNF leads to an efficient algorithm.

\section{EXTENSION TO PARENT MULTILATTICES}

In this section, we give an explicit description of the algorithm. We extend the original algorithm of Ref. 1 to include enumeration of structures derived from a multilattice. The original algorithm only addressed the case of parent lattices that were Bravais lattices. Part 1 of the extended algorithm (generating the superlattices) is identical to the first part of the original algorithm. The discussion of the previous section described part 2 of the algorithm-how equivalent labelings of a multilattice can be treated as members of a permutation group.

(1) Generate all derivative superlattices that are symmetrically distinct.

(a) Generate all possible superlattices of size $n$ (for each positive integer $n$ ) by constructing all integer matrices $\mathbb{H}$, in HNF, with determinant $|\mathbb{H}|=n$. This eliminates the possibility of listing multiple bases for the same lattice. A matrix in $\mathrm{HNF}$ is a lower triangular integer matrix of the form:

$$
\left(\begin{array}{lll}
a & 0 & 0 \\
b & c & 0 \\
d & e & f
\end{array}\right)
$$

subject to the conditions that $0 \leq b<c, 0 \leq d<f, 0 \leq e<f$, and $a \cdot c \cdot f=n=|H|$. Because the HNF is a unique representation of an integer lattice, the complete list of superlattices is found by listing (as their bases) all matrices of the form $\mathbb{B}$ $=\mathrm{AH}$. Here, the columns of $\mathrm{A}$ are the basis vectors of the parent lattice, $\mathbb{H}$ is an integer matrix in $\mathrm{HNF}$, and the column vectors of $B$ are the basis vectors of the superlattice.

If our multilattice is hcp, for example, with parent lattice $L$ generated by the columns of

$$
A=\left(\begin{array}{ccc}
1 & 1 / 2 & 0 \\
0 & \sqrt{3} / 2 & 0 \\
0 & 0 & \sqrt{8 / 3}
\end{array}\right),
$$

and displacement set

$$
D=\left\{\left(\begin{array}{l}
0 \\
0 \\
0
\end{array}\right),\left(\begin{array}{c}
1 / 2 \\
\sqrt{3} / 2 \\
\frac{1}{2} \sqrt{8} / \sqrt{3}
\end{array}\right)\right\},
$$

and if $n=2$, for example, we multiply $\mathrm{A}$ by each of the seven Hermite Normal Form matrices of determinant 2,

$$
\begin{gathered}
\left(\begin{array}{lll}
2 & 0 & 0 \\
0 & 1 & 0 \\
0 & 0 & 1
\end{array}\right),\left(\begin{array}{lll}
1 & 0 & 0 \\
0 & 2 & 0 \\
0 & 0 & 1
\end{array}\right),\left(\begin{array}{lll}
1 & 0 & 0 \\
1 & 2 & 0 \\
0 & 0 & 1
\end{array}\right),\left(\begin{array}{lll}
1 & 0 & 0 \\
0 & 1 & 0 \\
0 & 0 & 2
\end{array}\right), \\
\left(\begin{array}{lll}
1 & 0 & 0 \\
0 & 1 & 0 \\
1 & 0 & 2
\end{array}\right),\left(\begin{array}{lll}
1 & 0 & 0 \\
0 & 1 & 0 \\
0 & 1 & 2
\end{array}\right),\left(\begin{array}{lll}
1 & 0 & 0 \\
0 & 1 & 0 \\
1 & 1 & 2
\end{array}\right),
\end{gathered}
$$

obtaining bases of the seven possible superlattices of index $n=2$. 


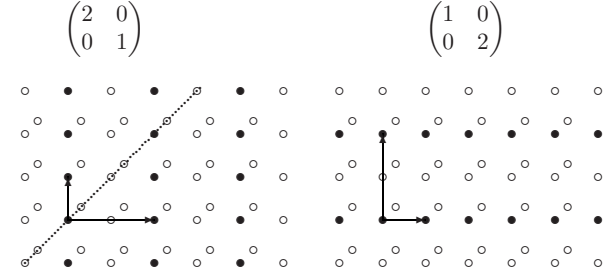

FIG. 5. Two equivalent multilattices. Though the bases are different, the first multilattice can be transformed into the second by a reflection about the line $x-y=0$. (Note that a $90^{\circ}$ rotation is not a symmetry of the multilattice but the reflection is.)

(b) Eliminate bases that represent rotationally equivalent superlattices even though the vectors are not identical. Some superlattices may be equivalent by rotations or reflections that are part of the symmetry group ${ }^{7}$ of the parent lattice, as shown in Fig. 5. Two bases $\mathbb{B}_{1}$ and $\mathbb{B}_{2}$ represent equivalent lattices by an orthogonal symmetry $R$ of the parent lattice if and only if $\mathrm{B}_{2}^{-1} R \mathrm{~B}_{1}$ is an integer matrix.

In the case of hcp, index 2 , for example, multiplying $A$ by

$$
H=\left(\begin{array}{lll}
2 & 0 & 0 \\
0 & 1 & 0 \\
0 & 0 & 1
\end{array}\right)
$$

gives a superlattice, which is the same as multiplying $\mathrm{A}$ by

$$
H=\left(\begin{array}{lll}
1 & 0 & 0 \\
0 & 2 & 0 \\
0 & 0 & 1
\end{array}\right)
$$

and then reflecting the entire multilattice through a vertical plane $30^{\circ}$ away from the $x$ axis. In other words, they are equivalent via a symmetry of the multilattice, so only one of the two needs to be included in our list.

(2) Construct a finite structure to represent the multilattice (for each remaining superlattice). This structure will be a Cartesian product $D \times G$, where $D$ is the set of fractional translations of the parent lattice $L$, whose union is $M$ (i.e., see Fig. 1 and the Appendix), and $G$ is a finite Abelian group.

(a) Construct the quotient group $G=L / L^{\prime}$, where $L$ is the parent lattice and $L^{\prime}$ the superlattice. Given the basis matrix $\mathrm{AH}$ for $L^{\prime}$, convert $H$ to a $\mathrm{SNF}$ : $U H V=S$, where $U$ and $V$ are integer matrices with determinant \pm 1 .

$$
\mathrm{S}=\left(\begin{array}{ccc}
s_{1} & 0 & 0 \\
0 & s_{2} & 0 \\
0 & 0 & s_{3}
\end{array}\right)
$$

is a diagonal matrix with positive integer entries and each diagonal entry divides the next one down. That is, $s_{2}$ is evenly divisible by $s_{1}$ and $s_{3}$ is evenly divisible by $s_{2}$. The quotient group $L / L^{\prime}$ is isomorphic to the group $G=\mathbf{Z}_{s_{1}}$ $\oplus \mathbf{Z}_{s_{2}} \oplus \mathbf{Z}_{s_{3}}$, where $\mathbf{Z}_{n}=\mathbf{Z} / n \mathbf{Z}$ represents the cyclic group of order $n$. This fact is central to the algorithm; it is the key that provides a mapping between equivalent labelings and members of the group $G$.

In the $n=2$ hcp example we have been using, all seven of the HNFs given in Step 1(a) have the same SNF:

$$
\left(\begin{array}{lll}
1 & 0 & 0 \\
0 & 1 & 0 \\
0 & 0 & 2
\end{array}\right)
$$

which corresponds to the quotient group $\mathbf{Z}_{2}=\{0,1\}$ (where the group operation on this set is addition modulo 2 ).

(b) Construct a map $h$ from the parent lattice onto the group $G$. Let $h: L \rightarrow G$, where

$$
h(x)=\left[U A^{-1} x\right]_{\mathrm{S}}
$$

and []$_{\mathrm{S}}$ means that every element of a column vector is to be reduced modulo the corresponding diagonal element of $\mathrm{S}$. Note that this map $h$ provides a correspondence between translates of $L^{\prime}$ and elements of $G$ (see the Appendix for greater detail).

(c) Define a map $\gamma$ from our multilattice onto the Cartesian product $D \times G$. Because every element $y \in M=D+L$ can be uniquely represented in the form $y=d+x$ for $d \in D$ and $x \in L$ (the parent lattice), we define

$$
\gamma(y)=\gamma(d+x)=(d, h(x)) .
$$

Two elements of the multilattice will have the same $\gamma$ image if and only if they differ by an element of the superlattice; i.e., if and only if they are forced to receive the same label in every possible labeling of the multilattice. So, labeling the elements of $D \times G$ is equivalent to labeling the multilattice in a manner that is periodic with respect to the superlattice. This is a key step in the algorithm because it simplifies the identification of duplicate labelings; via the map $\gamma$, equivalent labelings are represented by permutations of the $D \times G$ table.

In our ongoing $n=2$ hcp example [see Steps 1(a) and $1(\mathrm{~b})]$, we will have $G=\{0,1\}$ (since we noted that the quotient group is $\mathbf{Z}_{2}$ ) and

$$
D=\left\{\left(\begin{array}{l}
0 \\
0 \\
0
\end{array}\right),\left(\begin{array}{c}
1 / 2 \\
\sqrt{3} / 6 \\
\frac{1}{2} \sqrt{8} / \sqrt{3}
\end{array}\right)\right\} .
$$

Thus the structure to be labeled will be the four elements of the Cartesian product $D \times G$, namely,

$$
\begin{aligned}
& \left(\left(\begin{array}{l}
0 \\
0 \\
0
\end{array}\right), 0\right), \quad\left(\left(\begin{array}{l}
0 \\
0 \\
0
\end{array}\right), 1\right), \quad\left(\left(\begin{array}{c}
1 / 2 \\
\sqrt{3} / 6 \\
\frac{1}{2} \sqrt{8} / \sqrt{3}
\end{array}\right), 0\right), \\
& \text { and }\left(\left(\begin{array}{c}
1 / 2 \\
\sqrt{3} / 6 \\
\frac{1}{2} \sqrt{8} / \sqrt{3}
\end{array}\right), 1\right) .
\end{aligned}
$$

Labeling these four pairs with four labels is equivalent to labeling our multilattice periodically with respect to the given superlattice. This equivalence [given by $\gamma$ in Step 2(c)] enables us to label the product instead of directly labeling the lattice, the former being much simpler algorithmically. The equivalence is nontrivial and is derived in the Appendix.

(3) Determine the symmetries of our multilattice and represent these as permutations of the structure $D \times G$. 
(a) Each element of the parent lattice (thought of as a vector in space) is also a translational symmetry of the multilattice, and is represented by adding a fixed element of $G$ to every other element of the group $G$. Thus the corresponding permutation of the structure $D \times G$ is simply an operation on the right-hand coordinate, leaving the left coordinate fixed. There is one such permutation for every element of $G$, and these represent all possible translational symmetries of the multilattice.

(b) Nontranslational symmetries are of the form $f(x)$ $=N x+t$, where $N$ is an orthonormal matrix representing an orthogonal symmetry of $L$ and of $L^{\prime}$, and $t$ represents a translation by adding the element $t$. For each orthonormal symmetry $N$ of the parent lattice $L$, determine whether there is a corresponding symmetry $f_{N}(x)=N x+t$ of the multilattice $M$ $=L+D$. If so, there will be many such symmetries, corresponding to values of $t$ differing by elements of the parent lattice $L$, but we need only one such symmetry (since we are going to compose them with translations anyway). If $N$ is also a symmetry of the superlattice $L^{\prime}$, then $f(x)$ simply rearranges the translates of $L^{\prime}$ within $M$; hence, $f(x)$ induces a permutation $\Gamma_{N}$ of the structure $D \times G$. See the Appendix for a complete description of how to compute this permutation.

(c) The set of compositions (a nontranslational symmetry followed by a translation) of the preceding two types of permutations comprises the group of all possible symmetryinduced transformations of the structure $D \times G$.

(4) Represent all possible labelings of the structure $D$ $\times G$, and use the permutation group above to eliminate redundancy.

(a) We use the set $\mathcal{L}=\left\{0,1, \ldots, k^{m}-1\right\}$, where $m$ is the cardinality of $D \times G$, to index the set of all possible labelings. Here, $k$ is the number of labels; that is, in the case of a binary labeling, $k=2$, for a ternary, $k=3$, etc. Permutations of $D \times G$, as described above, induce permutations of the set $\mathcal{L}$, allowing us to eliminate symmetrically equivalent labelings.

(b) We eliminate labelings that we call superperiodic (corresponding to labelings that could be formed from smallerindex superlattices, that is, nonprimitive unit cells), and labelings that are equivalent by simply renaming the labels. For an example, see Fig. 7 in Ref. 1.

\section{ENUMERATION OF HCP-DERIVED SUPERSTRUCTURES}

As discussed above, the enumeration of derived superstructures consists of two steps. The first is to enumerate all symmetry-inequivalent superlattices; the second is to enumerate all labelings. Table I shows the number of superlattices (HNFs) for an hcp parent lattice and compares the number to that of other common parent lattices. The hcp parent is different from the rest: it is a multilattice, whereas the others are simple (Bravais) lattices.

The rotational and translation symmetries of a parent lattice influence the number of superlattices that can be derived from it. The number of superlattices for a given index $n$ will be lowest for parent lattices with higher symmetry. This is why the cubic cases have fewer superlattices than the hcp parent-the cubic cases have 48 operations in the point
TABLE I. Number of symmetrically distinct superlattices (HNFs) for several different parent lattices as a function of the index size $n$. The hexagonal case has more distinct superlattices than the cubic cases of the same index. This is because the cubic cases have higher symmetry. The higher the symmetry of the parent lattice, the fewer distinct superlattices can be derived from a parent. Note that the number is not the same for fcc/bcc and sc.

\begin{tabular}{lcccc}
\hline \hline \multirow{2}{*}{$\begin{array}{l}\text { Index } \\
n\end{array}$} & \multicolumn{4}{c}{ No. of superlattices } \\
\cline { 2 - 5 } & Hex & fcc/bcc & sc & Tetragonal \\
\hline 2 & 3 & 2 & 3 & 5 \\
3 & 5 & 3 & 3 & 5 \\
4 & 11 & 7 & 9 & 17 \\
5 & 7 & 5 & 5 & 9 \\
6 & 19 & 10 & 13 & 29 \\
7 & 11 & 7 & 7 & 13 \\
8 & 34 & 20 & 24 & 51 \\
9 & 23 & 14 & 14 & 28 \\
10 & 33 & 18 & 23 & 53 \\
\hline \hline
\end{tabular}

group, whereas hcp has only 24. Curiously, the simple-cubic (sc) and fcc/bcc cases do not have the same number of superlattices even though they have the same rotational symmetry group. The difference must be because the translational symmetries are not the same, but this leaves one to wonder why bcc and fcc have the same number. No doubt, this is an "accident" that occurs because of the reciprocal relationship of the bcc and fcc lattices.

The second step of enumerating the derivative lattices is to generate all the symmetrically distinct labelings for each superlattice. Table II contrasts the number of labelings for fcc and hcp cases listed two ways: (1) as a function of index and (2) as a function of the number of sites per cell. We see a big difference between simple lattices and multilattices. Because multilattices have multiple sites for each parent cell, there are far more combinatorial possibilities for the labelings of a multilattice. In the case of hcp, a superlattice with

TABLE II. Number of symmetrically distinct labelings of hcpand fcc-derived superlattices. For the same index, hcp has far more labelings, but this is merely a consequence of the fact that hcp has twice as many sites to label for the same index. In comparing the number of labelings as a function of the number of sites (right-hand side of the figure), fcc has more.

\begin{tabular}{lccccc}
\hline \hline$n$ & hcp & fcc & No. of sites & hcp & fcc \\
\hline 2 & 7 & 2 & 2 & 1 & 2 \\
3 & 30 & 3 & 3 & - & 3 \\
4 & 163 & 12 & 4 & 7 & 12 \\
5 & 366 & 14 & 5 & - & 14 \\
6 & 2613 & 50 & 6 & 30 & 50 \\
7 & 5268 & 52 & 7 & - & 52 \\
8 & 42901 & 229 & 8 & 163 & 229 \\
9 & 119528 & 252 & 9 & - & 252 \\
10 & 662193 & 685 & 10 & 366 & 685 \\
\hline \hline
\end{tabular}




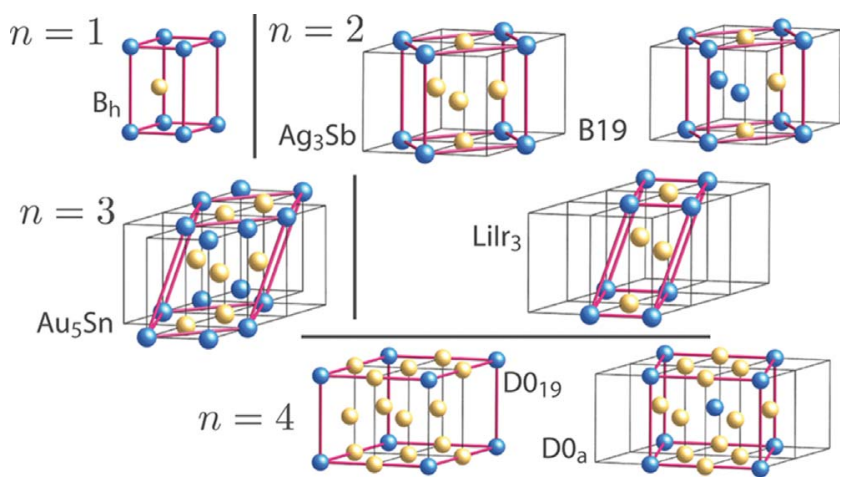

FIG. 6. (Color online) Seven binary hcp-derived superstructures (Ref. 5). These structures are all of the experimentally known hcpderived structures with eight atoms/cell or less. $B_{h}$ (WC) has in index of $n=1 ; B 19$ (AuCd), $\mathrm{Ag}_{3} \mathrm{Sb}$, and $\mathrm{LiI}_{3}$ all have index $n=2$; the $\mathrm{Au}_{5} \mathrm{Sb}$ structure has $n=3$; and the last two, $D 0_{19}\left(\mathrm{Ni}_{3} \mathrm{Sn}\right)$ and $D 0_{a}\left(\beta \mathrm{Cu}_{3} \mathrm{Ti}\right)$, have index $n=4$. In the pictures, the red (thick) lines indicate the primitive unit cells; the thin black lines are the underlying parent hcp cells. For an index of $n \leq 4$, our algorithm finds 201 hcp-derived structures, but only these 7 have been observed.

an index $n=2$ has four sites to be labeled whereas an fcc superlattice with the same index has only two.

On the other hand, for the same number of sites, the fcc case has more distinct labelings. This may seem counterintuitive because fcc has a higher symmetry than hcp. The difference is accounted for not by the symmetry, but by the number of superlattices of each. For a given number of sites, the index of the hcp case is half that of the fcc case (i.e., the fcc case has a larger number of superlattices than the hcp case because its index is greater). This effect more than compensates for the higher symmetry; the number of superlattices is a rapidly increasing function of the index (see Table I).

\section{APPLICATION TO HCP-BASED ALLOYS}

Binary intermetallic compounds that are derivative superstructures of the parent hexagonal-close-packed structure seem to occur less frequently than derivative structures of the fcc or bcc parent cells. Figure 6 shows the known hepderived superstructures as listed in the compendium by Sluiter. ${ }^{5}$ For indices of $n=1-4$ (2-8 atoms/cell), there are only seven known derivative superstructures that appear experimentally. In contrast, for 1-8 atoms/cell, there are several dozen experimentally observed derivative superstructures for the bcc and fcc lattices. Are there new hcp derivative superstructures enumerated by our method that might appear experimentally?

Reference 8 claims that simple geometric arguments can be used to make qualitative statements about this question. In short, atomic configurations in a derivative supercell are more likely if they are "unrandom," that is, if the configuration is significantly different from a random arrangement. The argument compares the supercell labeling with a perfectly random superstructure at the same concentration. The deviation from the random case was measured by looking at

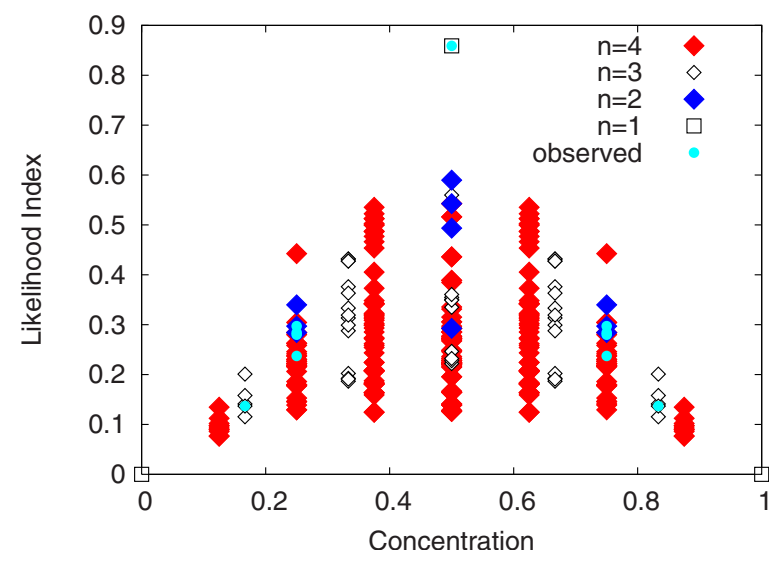

FIG. 7. (Color online) The likelihood measure of hcp derivative structures (index $n=1-4$ ). A few enumerated structures have higher measures than the seven experimentally observed structures.

pair correlations. Applying the same concept here, ${ }^{9}$ we rank the enumerated hcp structures by likelihood measure, according to concentration (see Fig. 7). A few enumerated structures have higher likelihood measures than the seven experimentally observed structures. Such structures are good candidates for new intermetallic compounds and should be considered in constructing cluster expansions, high throughput searches, ${ }^{10}$ and $\mathrm{x}$-ray determination of unknown structures.

\section{SUMMARY}

We presented an algorithm for enumerating all the superstructures derived from a parent lattice. Although much more in-depth discussion of key parts of the algorithm was given in Ref. 1, this new work is more general-it applies also to the case where the parent lattice is a multilattice. The algorithm relies on a group-theoretic representation of the superstructures, utilizing the properties of integer matrices. The running time of the algorithm scales linearly with the number of unique structures, the best possible scaling for this type of problem.

The key idea of the algorithm is to provide a mapping from the geometric representation of multilattice labelings to labelings of a simple Cartesian product involving a finite group. Then, the equivalence of geometric structures can be represented simply as permutations of the Cartesian product. With this representation, reducing the set of all combinatorially possible labelings to those that are symmetrically unique is simple and efficient.

As an example, we applied the algorithm to the case of hcp-derived superstructures. Compared to the fcc case, there are far more possible labelings for the hcp case, despite the fact that more fcc structures have been observed experimentally. Using the idea of Ref. 8, we conjecture that a few new hcp-based intermetallic phases may be found. A FORTRAN95 implementation of the algorithm is available for free download. ${ }^{11}$

\section{ACKNOWLEDGMENTS}

G.L.W.H. gratefully acknowledges financial support from 
the National Science Foundation through Grant No. DMR0650406. The authors are grateful to Lance Nelson for making the images for Fig. 3.

\section{APPENDIX}

This appendix contains a more detailed mathematical exposition of the algorithm. We begin with a (full rank) lattice $L$ and a finite set $D$ of fractional translations (so called because they are typically presented as elements interior to the basis tile of $L$ ). We may include the origin as an element of $D$, however, so our multilattice $M$ is equal to the set $L+D$. We also assume that the presentation of $M$ has been chosen so that $L$ is the full group of translational symmetries of $M$.

Among the isometries of $\mathbf{R}^{3}$, let $\mathcal{S}$ be the subgroup consisting of those that are symmetries of $M$. These are all of the form $f(x)=N x+t$ (multiplication of column vector $x$ on the left by an orthonormal matrix $N$ and followed by a translation by vector $t$ ), where $N$ belongs to a special subgroup $\mathcal{N}$ of the orthogonal transformations of $\mathbf{R}^{3}$ that are symmetries of $L$. For each $N \in \mathcal{N}$, there exists a unique element $d_{N}$ in $D$ so that $f(x)=N x+t$ is a symmetry of $M$ if and only if $t$ is in the infinite set $L+d_{N}$. (Note: the requirement $d_{N} \in D$ depends on assuming that $D$ contains the origin.)

Now we introduce our superlattice $L^{\prime}<L$ (meaning it is a subgroup of $L$ ). Recall that the basis matrix for $L^{\prime}$ is $A H$, where $\mathrm{A}$ is the basis matrix for $L$ and $H$ is an integer matrix in Hermite normal form, with the associated Smith normal form matrix $\mathrm{S}=\mathbb{U H V}$. Thus $A H V$ is also a basis for $L^{\prime}$; a generic element of $L^{\prime}$ is $w=\mathrm{AHV} z$ where $z$ is a column of integers. Thus $\mathrm{UA}^{-1} w=S z$ if and only if $w \in L^{\prime}$. This provides the required homomorphism $h$ from $L$ onto the group $G=\mathbf{Z}_{S_{11}} \oplus \mathbf{Z}_{S_{22}} \oplus \mathbf{Z}_{S_{33}}$, with kernel $L^{\prime}$, as follows:

$$
h(w)=\left[\mathrm{UA}^{-1} w\right]_{\mathrm{S}},
$$

where []$_{\mathrm{S}}$ means we simply reduce the $i$ th entry in a column vector to its least the corresponding entry $S_{i i}$ in the SNF matrix S. In other words, if $C$ is a column of integers, then $(C)_{\mathrm{S}}$ is an element of $G$. Since $h$ is a homomorphism of groups, with kernel $L^{\prime}$, we know (First Homomorphism Theorem of group theory) that $G$ is isomorphic to the quotient group $L / L^{\prime}$ (whose elements are the translates of $L^{\prime}$ in $L)$.

Next we consider the effects of various symmetries on our structure $D \times G$. If $t \in L$, then the translational symmetry $y$ $\rightarrow y+t$ sends a multilattice element $y=d+x$ (where $d \in D$ and $x \in L)$ to $y^{\prime}=d+x^{\prime} \quad$ where $x^{\prime}=x+t \in L$. Thus $\gamma\left(y^{\prime}\right)$ $=\left(d, h\left(x^{\prime}\right)\right)=(d, h(x)+h(t))=\gamma(y)+(0, h(t))$, where $\gamma$ is the projection of $M$ into the Cartesian product $D \times G$. In other words, a translational symmetry is simply represented by adding a constant group element to the second entry in $D$ $\times G$ as follows:

$$
\pi_{t}(d, g)=\left(d, g+g_{0}\right)
$$

where $g_{0}=h(t)$. Note that every element of $G$ can be the additive constant $g_{0}$, for some $t$, so the effects of translations on $M$ are completely described by all maps of the form $\pi(d, g)=\left(d, g+g_{0}\right)$.

What about nontranslational symmetries? If $y=d+x$ again, the symmetry $f_{N}(y)=N y+d_{N}=N x+N d+d_{N}=N x+d_{N, d}$ $+t_{N, d}$, where $d_{N, d}$ and $t_{N, d}$ are elements of $D$ and $L$, respectively, which depend only on $N$ and $d$. These elements $\left(d_{N, d}\right.$ and $\left.t_{N, d}\right)$ can be computed ahead of time. Thus, the symmetry $f_{N}$ maps an element $y=x+d$, with $\gamma(y)=(d, h(x))$, to the element $\quad y^{\prime}=f(y) \quad$ with $\quad \gamma\left(y^{\prime}\right)=\left(d_{N, d}, h\left(N x+t_{N, d}\right)\right)$ $=\left(d_{N, d},\left(U A^{-1} N x\right)_{S}+\left(U A^{-1} t_{N, d}\right)_{S}\right)$. The element $d_{N, d}$ can be regarded as a permutation of the rows of $D \times G$, while the second entry can be regarded as a permutation on the group $G: h(x) \rightarrow\left(U A^{-1} N A U^{-1} h(x)\right)_{S}+\left(U A^{-1} t_{N, d}\right)_{S}$ (defined by $N$ and $d)$. We may drop the notation ( $)_{\mathrm{S}}$ henceforth by assuming that operations in the second coordinate of $D \times G$ will always take place within the group $G$. Thus $f_{N}$ induces the permutation

$$
\pi_{N}(d, g)=\left(d_{N, d},\left(U A^{-1} N A U^{-1}\right) g+\left(U A^{-1}\right) t_{N, d}\right) .
$$

The permutations $\pi_{t}$ and $\pi_{N}$, as defined above, generate the full group of permutations induced on $D \times G$ by the symmetries $S$ of $M$. In fact, every such induced permutation is of the form $\pi_{t} \circ \pi_{N}$ for some $t$ and $N$. By constructing the finite group of symmetries acting upon the finite set $D \times G$, we have effectively described all possible isometries acting upon the periodic labelings of the multilattice $M$.

Now we may represent periodic labelings of the multilattice as $m$-digit numbers to base $k$, where $k$ is the number of available labels and $m$ is the cardinality of $D \times G$. In other words, each element in the set $\mathcal{L}=\left\{0,1,2, \ldots, k^{m}-1\right\}$ represents a labeling of the elements of $D \times G$ (equivalently, a periodic labeling of the multilattice), and all possible periodic labelings are represented uniquely in this way. For convenience, we index the elements of $G$ and $D$ so $G$ $=\left\{g_{0}, g_{1}, \ldots, g_{n-1}\right\}$ (where $\left.n=|G|\right)$ and $D=\left\{d_{0}, d_{1}, \ldots, d_{j}\right\}$. Given $x \in \mathcal{L}$, expand $x$ to base $k$ so $x=\sum_{i=0}^{m-1} x_{i} k^{i}$. This element $x$ then corresponds to the labeling, which sends each element $\left(d_{i}, g_{j}\right) \in D \times G$ to the label $x_{i n+j}$.

Now a permutation $\pi$ of $D \times G$ can be written as $\pi\left(d_{i}, g_{j}\right)=\left(d_{\alpha(i, j)}, g_{\beta(i, j)}\right)$ (using functions $\alpha$ and $\beta$ to represent $\pi)$. Then, composing $\pi$ with the labeling defined by $x$ gives the labeling defined by $y$ where $y_{i n+j}=x_{\alpha(i, j) n+\beta(i, j)}$ or $y=\sum x_{\alpha(i, j) n+\beta(i, j)} k^{i n+j}$. Thus a permutation $\pi$ on $D \times G$ induces a permutation of the set $\mathcal{L}$ of labelings. We now assign a flag to each element of $\mathcal{L}$ and then progressively turn off these flags as we apply the permutations to determine which labelings are equivalent to ones already counted. 
${ }^{1}$ G. L. W. Hart and R. W. Forcade, Phys. Rev. B 77, 224115 (2008).

${ }^{2}$ D. Lerch, O. Wieckhorst, G. L. W. Hart, R. W. Forcade, and S. Müller, Modell. Simul. Mater. Sci. Eng. 17, 055003 (2009).

${ }^{3}$ In the mathematical literature, and in some of the crystallography literature, these derivative lattices are referred to as sublattices. We prefer this nomenclature, which is more correct from a group theory point of view, but we follow the nomenclature typically seen in the physics literature where a lattice (or a structure) whose volume is larger than that of the parent is referred to as a superlattice (or a superstructure).

${ }^{4}$ In practice, the atoms in a derivative structure do not lie precisely on the lattice points of the parent lattice, but the experimental structure can be readily associated with the ideal structure enumerated by this algorithm.

${ }^{5}$ M. H. F. Sluiter, Phase Transitions 80, 299 (2007).

${ }^{6}$ The idea of using the HNF to enumerate superlattices is essentially the work of Santoro and Mighell (Refs. 12 and 13).

${ }^{7}$ The idea of breaking the various cases down by Smith normal form was introduced in Ref. 14. The combinatorial approach to enumerating the nonequivalent sublattices for a specific point symmetry (by another standard technique, Burnside's Lemma) was introduced in Ref. 15. Our algorithm has the advantage of yielding the actual structures of each derivative structure, while the combinatorial techniques in these references provide the numbers of structures a correct algorithm must produce.

${ }^{8}$ G. L. W. Hart, Nature Mater. 6, 941 (2007).

${ }^{9}$ Mathematically, the likelihood measure is just the cumulative difference between the average pair correlations, $\bar{\Pi}$, for the structure and the correlations of a purely random case. That is,

$$
l=\sum_{i}^{\text {pairs }}\left|\bar{\Pi}_{i}-\bar{\Pi}_{\text {random }}\right| .
$$

Such a measure is concentration dependent because the $\Pi$ 's are, so $l$ is also concentration dependent. Reference 8 compared $l$ independent of the concentration because only a small number of enumerated structures were considered. In this case, it is more appropriate to compare the likelihood measures as a function of $x$. A final note, in defining the pairs associated with a given $\bar{\Pi}$, pairs that were symmetrically distinct but had the same length were taken together. So, in hcp, for example, the six in-plane nearest-neighbor pairs and the six nearest-neighbor out-of-plane pairs were taken together, yielding a single $\bar{\Pi}$.

${ }^{10} \mathrm{~S}$. Curtarolo, D. Morgan, and G. Ceder, CALPHAD: Comput. Coupling Phase Diagrams Thermochem. 29, 163 (2005).

${ }^{11}$ See EPAPS Document No. E-PRBMDO-77-001822 or enum at sourceforge.net to download the code. For more information about EPAPS, see http://www.aip.org/pubservs/epaps.html

${ }^{12}$ A. Santoro and A. D. Mighell, Acta Crystallogr., Sect. A: Cryst. Phys., Diffr., Theor. Gen. Crystallogr. 28, 284 (1972).

${ }^{13}$ A. Santoro and A. D. Mighell, Acta Crystallogr., Sect. A: Cryst. Phys., Diffr., Theor. Gen. Crystallogr. 29, 169 (1973).

${ }^{14}$ J. S. Rutherford, Acta Crystallogr., Sect. A: Found. Crystallogr. 51, 672 (1995).

${ }^{15}$ J. S. Rutherford, Acta Crystallogr., Sect. A: Found. Crystallogr. 48, 500 (1992). 